\title{
Transforming Curriculum Development: Putting an entire institution on line for the benefit of students, faculty, the professions and industry.
}

\author{
Shirley A. Holloway \\ The Northern Alberta Institute of Technology
}

\begin{abstract}
In response to current trends in post-secondary education, the Northern Alberta Institute of Technology (NAIT) in Edmonton, Alberta has embarked on an institute-wide initiative dubbed "LOGging Our Curriculum." The goal of the project is to create outcomes-based, modularized, digital curriculum housed in a database accessible to NAIT instructors via the Internet. The project is designed to assist NAIT to become more responsive to the fast changing requirements of industry; to provide instructors with shared curriculum development tools and resources; and to provide students with more learner-centered learning materials that adhere to a systematic instructional design model. The database created is the intellectual capital of the institution.

The segmentation of curriculum into modules (rather than courses) allows for effective and efficient redesign of curriculum and the customization of training. It also provides the foundation for Prior Learning Assessment and Recognition (PLAR), articulation between programs at NAIT and at other institutions, validation of curriculum with industry and facilitation of the accreditation process.
\end{abstract}

Rather than produce content-rich modules, the decision was made at the outset to produce Learning Outcome Guides (known as LOGs). A LOG is a module that does not (necessarily) embed lecture content, but instead includes pointers to content and activities. A LOG incorporates outcome-based language and instructional design based on Kolb's Experiential Learning Model. Faculty who decide to embed or link to lecture content, notes, audio or video in their LOGs may do so if they wish.

To assist program staff to "log" their curriculum by preparing Learning Outcome Guides, NAIT has developed a web-based curriculum development application called MERLIN. This guides instructors by providing online instructional design information to assist in writing learning outcomes and objectives, preparing the LOGs, creating courses, printing student materials and ultimately moving those materials to WebCT. MERLIN allows program areas to search for existing curriculum materials within the LOGging Database and use those materials as needed.

A companion project "VALIDATOR" is a web-enabled instrument for convenient validation of outcomes and is used for accreditation documentation and for professional and industrial validation.

Collecting curriculum into the LOGging database allows NAIT to respond to many challenges in a timely and effective manner. 
Introduction

In common with all academic institutions, The Northern Alberta Institute of Technology (NAIT) is facing the challenges of fulfilling its traditional role in education and training in face of an increasingly global economy that is being transformed by technological innovation. Shrinking government financial support poses a further challenge. The institution serves over 10,000 full time students as well as in excess of 50,000 part-time students who attend 195 separate and discrete programs 1,300 continuing education courses. As well, instruction and other forms of technical and educational assistance are currently offered in 29 other countries. As an undergraduate polytechnic institute NAIT is expected to provide job-ready graduates in engineering, computer, health and communication technologies and in business and hospitality. The required degree of sophistication of these graduates has increased with industry adoption of new techniques and there is a need to continually update both theory and practice expressed in the curriculum. The accreditation systems in Canada are rigorous and, since they are based on current industry standards, impose an additional stress on faculty and staff to maintain curriculum currency. Typically it has been left to individual departments and faculty to undertake almost perpetual curriculum redesign and implementation and to combine this activity with day to day teaching and their own professional development.

The keynote of NAIT's mission, academic plan and business plan is to promote student success in this new global economy. Whilst surveys show that NAIT is maintaining an excellent record of student retention, graduate placement and employer satisfaction, it is clear that true graduate success will depend on preparation for continued learning and development. There must be a change from curriculum that emphasizes the here and now of existing jobs to one which provides a platform for further learning. Traditionally the content of individual programs at NAIT has been set specifically for regional and national industry needs, with very little room for flexibility. Increasingly however NAIT is responding to demands for customized training that may utilize elements of more than one program as well as unique segments. Blending instructional elements from programs and courses prepared by individual (and sometimes idiosyncratic) instructors is not an easy task.

Another factor critical to student success and which is forcing change within the institution is the changing nature of student demands. Students are no longer prepared (or even able) to meet the normal program requirement of at least 30 hours of instruction inserted into a Monday to Friday, 9 to 5 schedule. Many institutions are moving to alternate delivery modes but rarely are efforts coordinated across the entire organization. Curriculum design and delivery often becomes inconsistent and, at worst, chaotic. NAIT intends to establish consistency in this area.

The LOGging Our Curriculum Project.

During the 1998-99 academic year, NAIT made the decision to embark upon an institution-wide coordinated curriculum project designed to meet the educational challenges of the new century. The need to normalize the process of curriculum renewal in face of variable industry dynamics has led to the establishment of an institutional curriculum database. The project was designed to meet a number of criteria and upon completion is expected to:

- create a pool of knowledge accessible to all faculty and students via the web 
- provide support for curriculum delivery through technology

- enhance student learning

- express the syllabus of each course or program in terms of measurable outcomes

- facilitate program validation and accreditation processes

The Learning Outcome Guides (LOGs)

In effect this digital curriculum database expresses the intellectual capital of the institution and underpins a strategic initiative to transform all NAIT curricula into outcomes-based modules. Each module relates to the achievement of a specific outcome and is termed a Learning Outcome Guide (LOG). The LOGs then are a product of the database, combining outcome-based language and instructional design based on Kolb's Experiential Learning Model. Arranging curriculum into smaller units allows for:

- Prior Learning Assessment and Recognition (PLAR)

- facilitation of remedial activities for students in academic difficulty

- articulation between programs within NAIT and other institutions

- efficient redesign of curriculum

- sharing of curriculum across the institution

- customization of training programs

- dynamic web-based curriculum delivery

- a uniform look and feel to all of NAIT programming

A LOG will contain the following components:

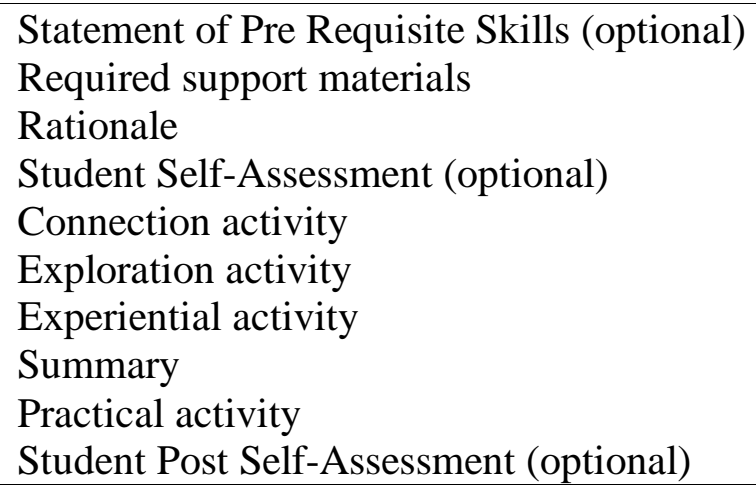

The LOG typically does not contain lecture material since the main purpose of the system is to facilitate classroom and laboratory activity rather than to create instant distant delivery materials. However, individual lecturers may elect to incorporate notes into their LOGs or use links to the web, a text or CD ROM. Versions of the LOGs created for distance delivery would be expected to be more complete in content and may include links to or embed video or audio lectures.

\section{Preparing and Working with a Digital Curriculum Database}

Typical instructors at NAIT are content experts in their field; however they are usually not pedagogical experts and, left to their own devices, may produce large quantities of technically valid content with little thought to instructional design. Furthermore, not all faculty members are 
familiar with the techniques required to prepare web-ready material. The LOGging our Curriculum project includes a provision to generate web pages consistent with the content available in the database. This ensures that a LOG that is printed, displayed on the web, or used to generate web pages will have a consistent "look and feel" and will ensure a pedagogically sound design.

\section{Web Page Creation}

NAIT has adopted WebCT as its standard web curriculum delivery tool. Integrating WebCT will spawn files that trick WebCT into thinking it created the course instead of the course being generated by the LOGging project software. Similarly, this course is then populated with web pages generated from the LOGging Our Curriculum database. Without the instructor's ever entering WebCT, a complete WebCT course is created. This approach not only achieves standardization but also absolves the instructor from learning HTML since entered text is automatically converted.

The Tools - MERLIN and MERLIN+.

MERLIN is a web based curriculum tool developed at NAIT. It has three components: MERLIN for instructors, MERLIN for students and MERLIN for NAIT.

- MERLIN is the web based curriculum tool to assist instructors in entering their LOGs with the help of a wizard or interactive on-line intelligent tutor.

- MERLIN is a web delivery vehicle for students. All LOGs will be available to students on the internet. The choice will be at the discretion of the program or instructor.

- MERLIN for NAIT is the user interface for the LOGging our Curriculum database. This will allow NAIT to have a catalogue of all LOGs.

Key features of MERLIN are:

- MERLIN's wizard provides online interactive assistance in all areas of LOG creation enabling the most inexperienced instructor to create well-designed properly formatted products for students.

- The LOG creation manager provides a comprehensive searchable catalog of LOGs that includes a Parent LOG and all versions nested beneath the parent. A Parent LOG is the version deemed to be the most applicable to the various courses or the most complete. Some faculty need to use a variant of the Parent for their particular interest group and will have that version nested. The LOG Creation Manager also allows Programs and courses to share/use LOGs easily.

- The Objective Manager assists instructors in the editing and preparation of Learning Outcomes statements and their associated objectives.

- Security is controlled at the program or instructor level.

- Web delivery and print capability of LOGs for student use is available.

- Summary report and course outlines are printable and allow for all curriculum information contained in the LOGs for any course to be in a useful format for students. 
MERLIN+.

Recently MERLIN+ has been added to the curriculum toolkit. It offers all the functionality of the existing MERLIN system along with several new features. All content will be entered in MERLIN+ via a WYSIWYG interface that allows cutting and pasting between Microsoft Word and MERLIN+ (while preserving formatting and tables), image inserting, and text formatting. MERLIN+ also allows users to print directly from its system instead of downloading the document as a word file.

MERIN+ addresses the need for a world-class software application. Currently we are exploring a model known in industry as the Application Service Provider (ASP) concept. NAIT clients would pay an annual subscription fee to use the MERLIN+ system. All content entered by the client would be housed separately from the NAIT curriculum database.

\section{Training for MERLIN}

Instructors are busy people and have little time to explore the technical niceties of MERLIN by themselves. Extensive training as groups, individually or on the web is available to all faculty and other staff (even the Deans have learned how to do a LOG). New NAIT faculty must enroll in an intensive program, Becoming a Master Instructor, before they commence teaching. This program has now incorporated MERLIN training so that all of their material is prepared in the LOGging format.

Issues of Curriculum Validation and Accreditation

All of NAIT's programs have Advisory Boards to assist faculty in maintaining curriculum currency. However, the range of the industry represented on the Advisory Boards is necessarily limited and a competency profile development (CPD) process (similar to a DACUM) was developed by NAIT for a broader consultation. Though this process has served NAIT well, it is somewhat cumbersome and the process of incorporating recommendations challenging. As part of the LOGging project, it was decided that an additional instrument, building on MERLIN, was required to streamline these processes.

VALIDATOR is NAIT's online curriculum validation system. It consists of three components: TRACKER, GENERATOR, Questionnaire Completion and Statistical Analysis.

- TRACKER is the system through which a program requests a validation study. It is also the system that maintains progress on the study.

- GENERATOR is the system that builds a questionnaire.

- Questionnaire Completion is the process of study participants taking a questionnaire online.

- Statistical analysis returns useful data from questionnaires.

Key features of VALIDATOR are:

- VALIDATOR provides a permanent system to record all results from a validation study.

- GENERATOR retrieves the outcomes and objectives from the MERLIN database as the questions on the questionnaire.

- Questionnaires completed online, with the data already saved in the database. No data entry from paper questionnaires is required.

- Production of useful results is automatic. 
The VALIDATOR has already been used in the development of two new programs during the last year and was invaluable in facilitating a rapid response to the needs of industry.

The LOGging database can be used to provide a list of outcomes for preparing materials for accreditation visits. Under development is a new tool (tentatively called Accreditation Assistant) to help faculty in this often onerous task. Advice is being sought from faculty in programs recently granted re-accreditation and from the accreditation teams involved before the instrument is finalized.

\section{Progress to Date}

At its inception in 1999, a target date for completion of the project was set at July 2002 (a period of three years). At the present time, this still seems a reasonable time frame. Almost all of the outcomes of all courses were listed in the database by June 2000. Some programs have completed their LOGging, many more will do so before June 2001 and the majority will have at least some of their courses on the web by that time.

Impact of LOGging our Curriculum on the Institution.

This project, though still not completed, is having a profound impact on students, faculty and the deployment of institutional resources. Students are particularly eager to adopt the new system. They said that the Learning Outcome Guide

- Assists me in preparing for class and reviewing for exams

- Provides "direction" to my studying

- Offers a uniform set of notes including all testable information

- Narrows in on what is really important

- Improves the learning process.

- I want to have all my courses in this format.

Instructors for the first time have the opportunity to share curriculum ideas across the institution. It is well known that all programs teach to many of the same learning outcomes. For example, learning outcomes associated with basic computer skills, team building, conflict management and basic laws of electricity are just a few of the learning outcomes critical to success in many of NAIT's programs. However, curricula to support those outcomes have traditionally been developed in isolation, program by program. Duplication of effort is rampant. For example the English department had more than eighty Effective Communications course outlines (containing hundreds of Learning Outcomes) developed for a variety of programs. After scrutiny of these outlines, faculty agreed upon twenty-one Learning Outcomes, many of which have now been incorporated into LOGs. As a shared resource available to all, the curriculum database allows faculty to both submit their ideas and draw on the curriculum development expertise of their colleagues. In addition, care has been taken to ensure there is room for individual faculty creativity in course delivery - especially as NAIT's reputation has been built on the excellence of its instructors and the "human touch". Instructors said

- Students appreciate the fact there is no mystery in the syllabus

- Well laid out information easily lends itself to a lesson plan format

- Each student can use a LOG differently, according to individual needs 
- For the first time I'm confident that the material I'm teaching is the most appropriate for my graduates to take into the workplace.

- The LOGging process supports team development of curriculum.

- This will really reduce the stress of preparing for accreditation visits.

The project has led the institution to re-examine allocation of instructional resources. On line students require the same or more care and attention than those in a traditional setting. Provision of online help and tutoring is crucial, as is the fostering of student to student interaction. Wireless technology has been introduced for students on the move, such as those in the forestry program. The latter can access program material and record experimental results while out in the field or even during travel to the site.

Statistics on adoption of the LOGging approach change by the day. However an indicator of success is that in December of 2000, over 6,000 students are registered in or taking a course or a modular component of a course through a web version of the LOG. This compares to 640 in the spring of 2000.

LOGging the Future.

In this new millennium, students will arrive at NAIT's doors (or electronic portal) with considerably different needs to those of the past. Most will be confident, technologically literate individuals who will demand learning that meets their personal requirements. LOGging Our Curriculum is just the first step in a process that will offer more on-demand learning for students and more personal choices. Other institutional resources must also be geared to the provision of better access to network resources, greater accessibility through electronic and face to face means to instructional staff, and greater acknowledgement of past learning through the implementation of systematic approaches to prior learning assessment and recognition.

The revolution in learning has already begun - innovation in academic environments is upon us. Institutes of technology are amongst those most directly affected by the revolution occurring in industry, yet by responding swiftly and effectively to these global economic forces they are positioned to be leaders in learning innovation.

\section{SHIRLEY HOLLOWAY}

Shirley Holloway is the Vice President, Academic and Student Services at the Northern Alberta Institute of Technology in Edmonton, Alberta, Canada. Dr. Holloway graduated in Pharmacy in England and received a M. Sc. from the Faculty of Medicine at the University of Western Ontario and a Ph.D. from the Department of Biology at York University in 1978. She is also certified as an applied science technologist and has been actively involved in many national, provincial and local initiatives in technical education. 